\title{
THE EFFECT OF HABITUATING VESTIBULAR AND OPTOKINETIC NYSTAGMUS ON EACH OTHER ${ }^{1}$
}

\author{
A. A. SKAVENSKI, ${ }^{2}$ S. M. BLAIR, ${ }^{3}$ AND G. WESTHEIMER
}

Department of Physiology-Anatomy, University of California, Berkeley, California 94720

\begin{abstract}
Currently, the vestibulo-ocular and optokinetic reflexes are both thought to require a velocity storage mechanism within their neural pathways. To test whether these storage mechanisms are shared by both reflexes, animals were given programs of stimulation known to change the status of the storage mechanism of one of the types of nystagmus. The other type of nystagmus was examined then to ascertain whether the characteristic of its velocity storage system had remained invariant. Horizontal eye movements of three macaque monkeys were recorded during post-rotatory nystagmus and optokinetic afternystagmus (OKAN) before and after 20 habituating exposures to either vestibular or optokinetic stimulation. Repetitive exposures to vestibular stimulation alone markedly reduced the time constant of post-rotatory nystagmus and this effect was accompanied only occasionally by a reduction in the time constant of OKAN. Repetitive exposure to optokinetic stimulation alone reliably reduced the time constant of OKAN but produced no reliable change in the time constant of post-rotatory nystagmus. These results suggest that the vestibulo-ocular and optokinetic reflexes do not share a single common velocity storage mechanism.
\end{abstract}

It is widely held that animals achieve fairly good gaze stability during natural head rotations by a combination of vestibulo-ocular and optokinetic reactions. The vestibulo-ocular reflex (VOR) employs neural correlates of head rotations derived from the semicircular canals to generate eye movements in the opposite direction. The optokinetic reflex (OKN) attempts to stabilize gaze using retinal image motion information. It has been proposed that these two reflexes share most neural processing elements except for their sensory inputs (e.g., see Raphan et al., 1977; Robinson, 1977a). A key feature of this suggestion is that both the optokinetic and vestibuloocular reflexes share a common velocity storage mechanism. The impetus for this suggestion is mainly one of parsimony. Namely, a velocity storage mechanism is needed to model the slow build-up of nystagmus during optokinetic stimulation as well as the persistence of that nystagmus in homogeneous visual fields after stimulation is stopped (Collewijn, 1972; Cohen et al., 1977). Inclusion of a velocity storage mechanism in the VOR pathway

${ }^{1}$ This research was supported by the National Eye Institute, United States Public Health Service under Grant EY-00592 and was carried out while Alex Skavenski was on sabbatical leave from the Department of Psychology, Northeastern University, Boston.

${ }^{2}$ To whom correspondence and reprint requests should be addressed at Department of Psychology, 234 NI, Northeastern University, Boston, MA 02115.

${ }^{3}$ Current address: Naval Regional Medical Center, Oakland, CA 94627. also provides a convenient basis for the observation that, during a constant velocity spin in total darkness, vestibular nystagmus persists much longer than the spin is signaled by the canals or peripheral nerve (Buettner et al., 1978; Fernandez and Goldberg, 1971; Skavenski and Robinson, 1973). Since a velocity storage mechanism is required by both reflexes, it may be argued that the same mechanism may serve both. This view is partially supported by the finding of Cohen et al. (1973) that labyrinthectomy, a lesion confined to the vestibular sensory apparatus, abolished optokinetic afternystagmus. However, this finding supports the common storage mechanism hypothesis only if it can be assumed that loss of the tonic vestibular output directly disrupts the storage mechanism and not the coupling of its output to the final common oculomotor path. Other findings that seem to support the single storage mechanism hypothesis require similar assumptions (e.g., see discussions by Raphan et al., 1977; Robinson, 1977a, b; and Waespe and Henn, 1977). There is little direct evidence. The experiments reported here are intended to fill that gap.

In monkeys, a repetitive program of constant velocity spins and stops in alternating directions in a Bárány chair causes reduced persistence of per- and post-rotatory vestibular nystagmus in a Ganzfeld. In fact, the time constant of the nystagmus can be made to approach the time constant that Fernandez and Goldberg (1971) have reported for the squirrel monkey's semicircular canals (about 6 sec; Blair and Gavin, 1979). In the metaphor of Figure 1, this effect, habituation, is equivalent to reducing 


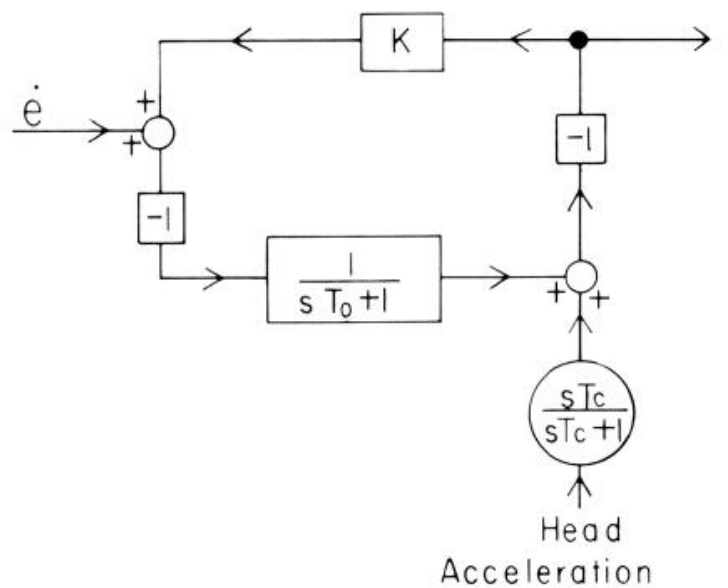

Eye Velocity

Figure 1. A simplified model (derived from Robinson, 1977a) illustrating one way eye velocity may be determined from optokinetic and vestibular stimulation. Retinal image slip velocity $(\dot{e})$ and head velocity signals from the canals both enter a positive feedback loop containing a gain element $(K)$ and a velocity storage mechanism (represented by $1 / s T_{0}+1$ ). The time constant of eye velocity is related to $K$ and the time constant of the velocity storage mechanism $\left(T_{0}\right)$ by $T=T_{0} / 1$ $-K(0<K<1)$ for optokinetic afternystagmus and also for vestibular nystagmus when $T_{0}$ is close to the value of the semicircular canal time constant $T_{c}$. All variables are in Laplace transform notation. These solutions do not include peripheral eye mechanics nor a neural integrator known to lie in the pathway of VOR peripheral to the site where eye velocity is determined (Skavenski and Robinson, 1973). See Robinson (1977a) for additional details on the solution of this model and its implications.

markedly the effect of the feedback pathway containing the velocity storage mechanism on the nystagmus; i.e., $K$ is made to approach zero. Such a reduction in the amount of feedback also would reduce markedly the effect of the velocity storage mechanism of $\mathrm{OKN}$ if it is identical with that of VOR. Note, the question of whether the same velocity storage mechanism is concerned with the maintenance of smooth velocity in both the optokinetic and vestibular systems exists for several plausible models that have been found adequate for the task of visualizing possible modes of operation of the circuits which are generally thought of as providing the regenerating outputs of eye velocity (e.g., Raphan et al., 1977; Robinson, 1977a). We simply present one example to illustrate the question which we have addressed: If there is only one velocity storage mechanism, then once its properties have been modified, such modification should manifest itself in all uses to which the mechanism is put. The present experiment tests this point. Specifically, we will show that a repetitive program of head angular accelerations in a Ganzfeld which reduced the persistence of vestibulo-ocular responses also only occasionally reduced the persistence of optokinetic afternystagmus (OKAN). However, a repetitive program of optokinetic stimulations which reduced persistence in that system did not reduce persistence in VOR reliably. These findings suggest that a single shared velocity storage mechanism does not describe adequately the regeneration of eye velocity in VOR and OKAN.
To assess the status of the velocity storage mechanism associated with optokinetic reflex, we measured the velocity and persistence of optokinetic afternystagmus in a Ganzfeld, where foveal smooth pursuit movements are not an interacting factor.

\section{Materials and Methods}

Three male juvenile Macaca speciosa were subjects in these experiments. None had served in experiments before and they received no behavioral training. The stainless steel screws for use as a head restraint were implanted in the skull of each monkey using sodium pentobarbital as the anesthesia. Each animal was given daily $0.5-\mathrm{cm}^{3}$ injections of tylosin antibiotic for 5 days after surgery or whenever signs of infection developed around the screws.

Horizontal eye movements were recorded with DC electro-oculograms (EOG) by means of nonpolarizing bitemporal skin electrodes. At the beginning of each session, a calibration of the EOG recording system was accomplished by attracting the monkey's gaze through known angular deviations in the horizontal plane. Calibrations were checked repeatedly throughout the experiments by monitoring EOG output as the animal carefully tracked an object moving at known constant velocity through its oculomotor range. In final measurements, eye position sensitivity was about $2^{\circ}$ arc and bandwidth was DC to $750 \mathrm{~Hz}$.

Vestibular nystagmus was elicited by head angular velocity steps obtained by rotating the monkey around a vertical axis in a servo-controlled chair. The monkey's head was held in the normal upright position by bolts to

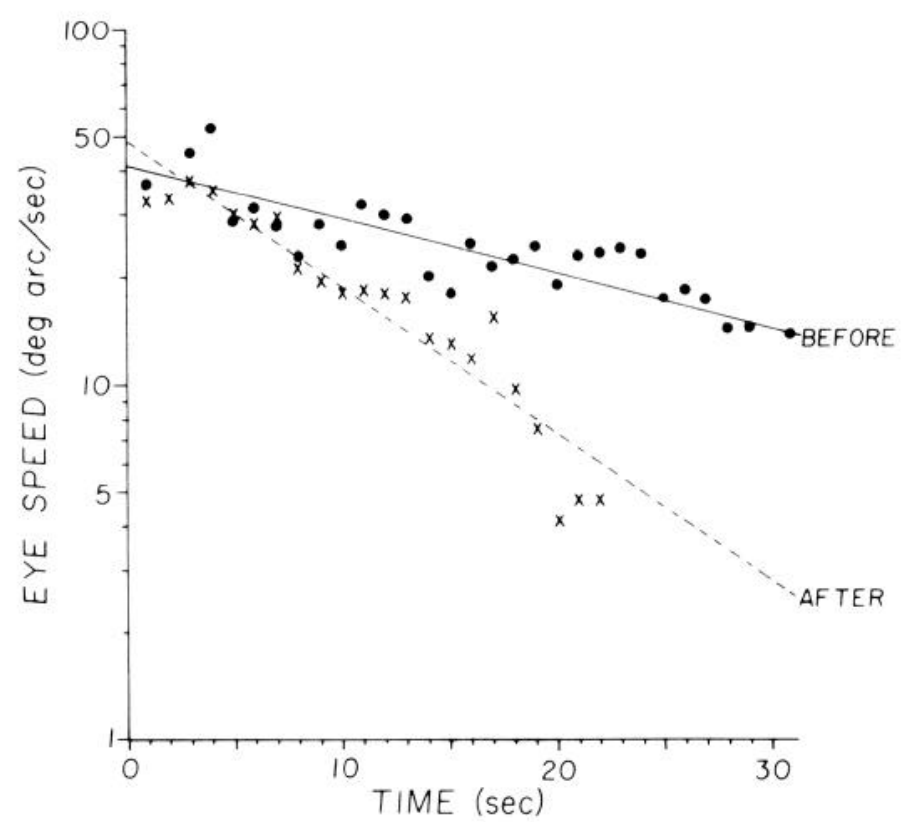

Figure 2. Representative time courses for the delay of eye speed during single tests of post-rotatory nystagmus made before $(\Theta)$ and after $(\times) 20$ VOR habituating spins. The monkey's head was abruptly decelerated from $80^{\circ}$ to $0^{\circ}$ arc $/ \mathrm{sec}$ at time $=0.0$ and the slow phase of nystagmus was to the right in both cases. Data were from monkey MS108's first session. Straight lines were fit by a least squares criterion. 
a frame attached to the chair and the body was supported by it. The angular position of the chair and hence, the monkey's head in space, was transduced by a high resolution, linear potentiometer. Both per- and post-rotatory nystagmus were examined only when the monkey viewed an illuminated Ganzfeld. Brightness of the Ganzfeld was choosen to maintain the animal's state of light adaptation for stability of the EOG recording.

Optokinetic nystagmus and afternystagmus were obtained as follows: A 1-m diameter drum with high contrast irregular features painted on its interior surface was rotated at constant velocity around the animal's stationary head. OKN was elicited by a 60 -sec exposure to the drum. OKAN was obtained by rapidly replacing the animal's visual world by a Ganzfeld. For this experiment, the Ganzfeld was a flat white hemicylinder with semicircular top and bottom plates that subtended the full visual field. A pulley apparatus permitted the experimenter to remove or replace it in front of the animal from outside the drum without stopping the drum's motion. In addition, the neutrality of the Ganzfeld was verified by placing it around the animals' heads after they spent a minimum of 5 min fixating objects in the room with both the visual environment and the animal stationary. Under these conditions, all of the monkeys showed eye movements comparable to those seen in total darkness.

Horizontal eye velocity was measured directly on the inkjet-writer records (Mingograf 800 ). Slow phase eye velocity was taken as the average velocity over a 200- msec interval that did not contain a quick phase. These measures were made at successive 1-sec intervals beginning 1 sec after the onset of post-rotatory vestibular nystagmus or OKAN and extended $20 \mathrm{sec}$ into the record. Exponential functions relating slow phase eye velocity to time then were fitted to each 30 -sec data set using a least squares criterion. A representative example of these data is shown in Figure 2. Our purpose here was to compare the persistence of nystagmus before and after habituation. A single time constant exponential clearly is suited to the description of OKAN which is the output of the storage mechanism alone and also for post-rotatory nystagmus when the dynamics of the cupula response and the velocity storage mechanism are fairly close (see solution in Fig. 1). The use of an exponential with a single time constant to describe the persistence of nystagmus in the present case was justified further by the goodness of fits that we obtained. Most of the coefficients of determination for linear fits on log eye velocity versus time coordinates (as in Fig. 2) were in excess of 0.8 and only a few ranged as low as 0.5 . The zero intercept of the exponential divided by head or drum velocity (gain) provided a measure of the strength of nystagmus. The zero intercept of the exponential is a better estimate of OKAN gain because the actual eye velocity may contain pursuit aftereffects that may persist up to $0.5 \mathrm{sec}$ after moving stimuli are removed from view.

Two experimental protocols were used. We began by habituating vestibular nystagmus and testing for a

TABLE I

Time constants of horizontal post-rotatory vestibular nystagmus (PRN) and optokinetic afternystagmus (OKAN) before and after three monkeys were subjected to 20 vestibular habituation spins in each of three sessions

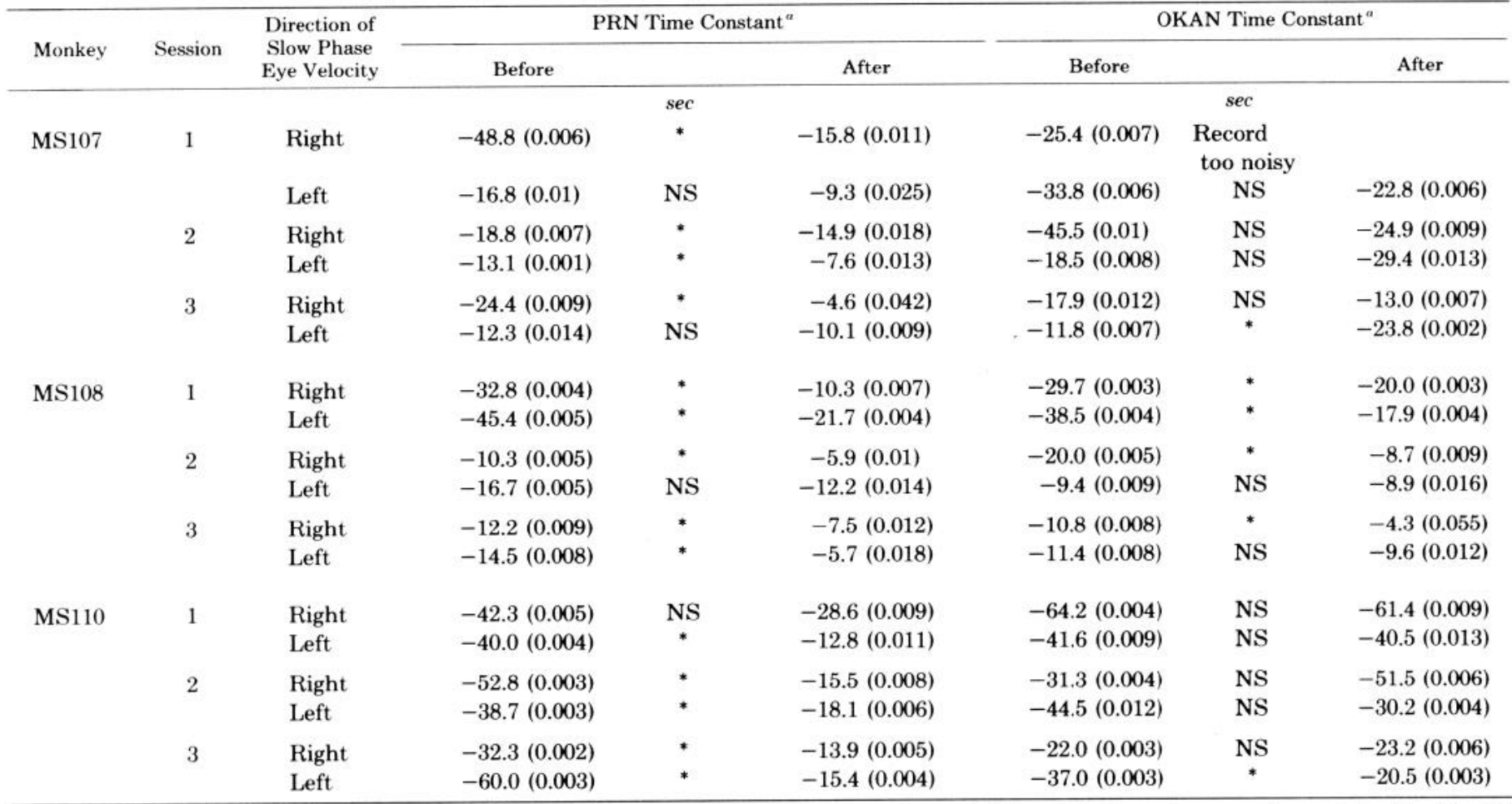

${ }^{a}$ Log standard error of the slope from which the time constant was computed (reciprocal of slope) is shown in parentheses. Asterisks identify those time constants that show statistically reliable changes $(p \leqslant 0.05)$ before and after habituation based on $t$ tests for the significance of the differences in the slopes of lines relating log eye velocity to time. NS indicates those before-after time constants that were not statistically reliably different. 
change in OKAN. For each monkey, there were at least three sessions. Each session began with a record of OKAN to the left and to the right following a 1-min exposure to optokinetic stimulation of $80^{\circ} \mathrm{arc} / \mathrm{sec}$. Then the animal was subjected to 20 to 40 constant velocity spins and stops in the illuminated Ganzfeld. Spins alternated in direction and each lasted 1 min or until perrotatory nystagmus completely subsided. The speed of

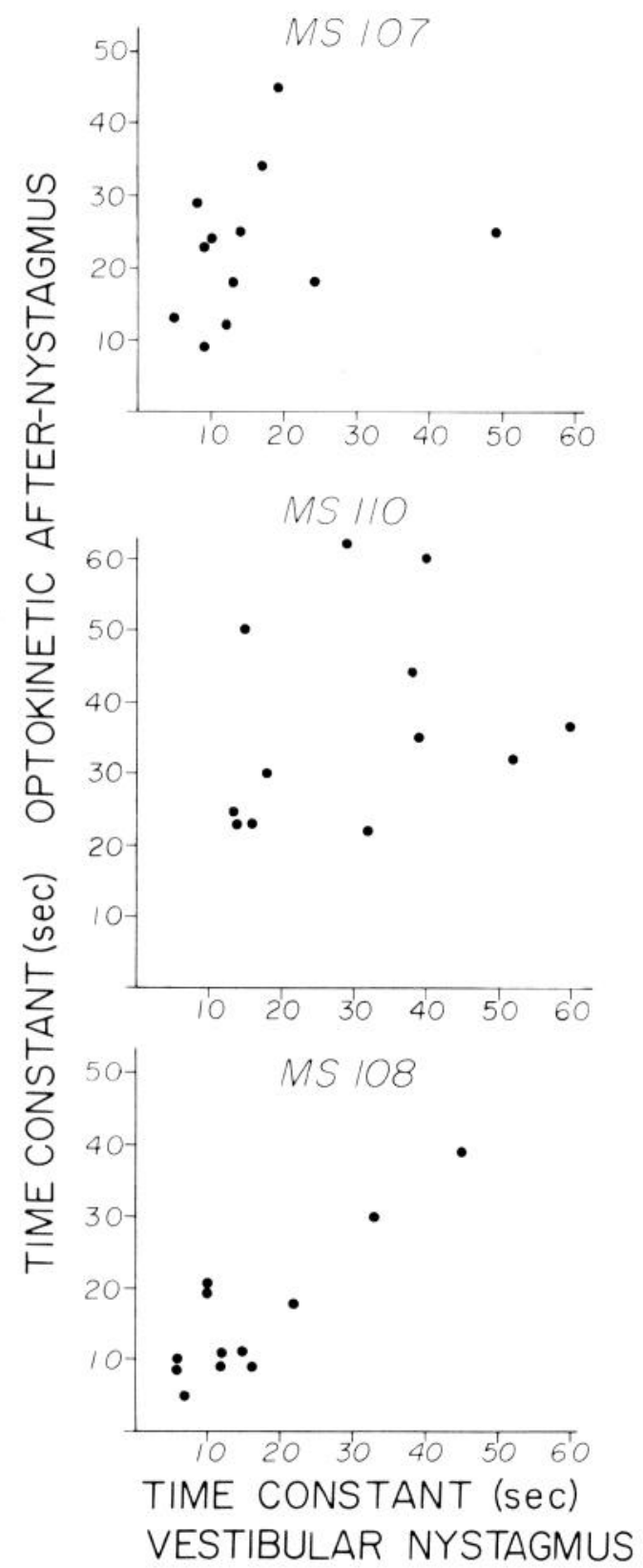

Figure 3. The relationship between the time constants of vestibular nystagmus and optokinetic afternystagmus when the persistence of both were measured at different states of habituation of the vestibulo-ocular reflex for three monkeys (MS107, MS110, and MS108). Each data point shows the time constant of VOR and OKAN for the same direction of the slow phase of nystagmus and the same state of VOR habituation. The scatter of the data was not reduced by separating left and right slow phase direction so no distinction was retained in this plot.

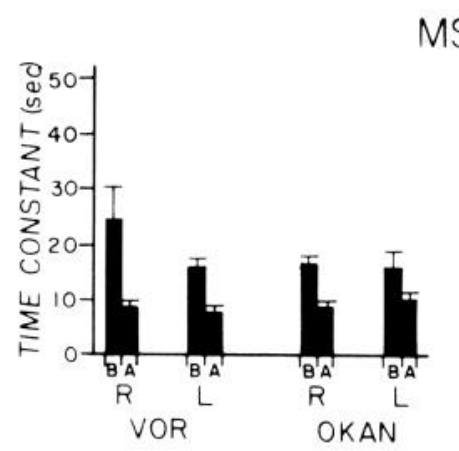

MS 107
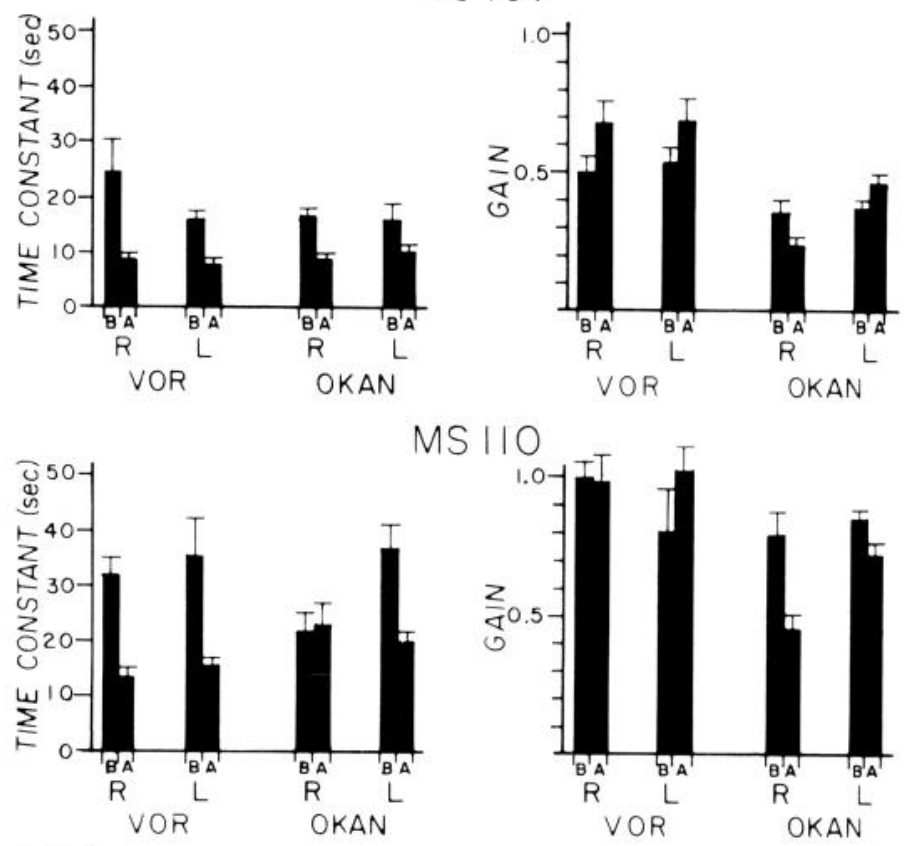

MS 110
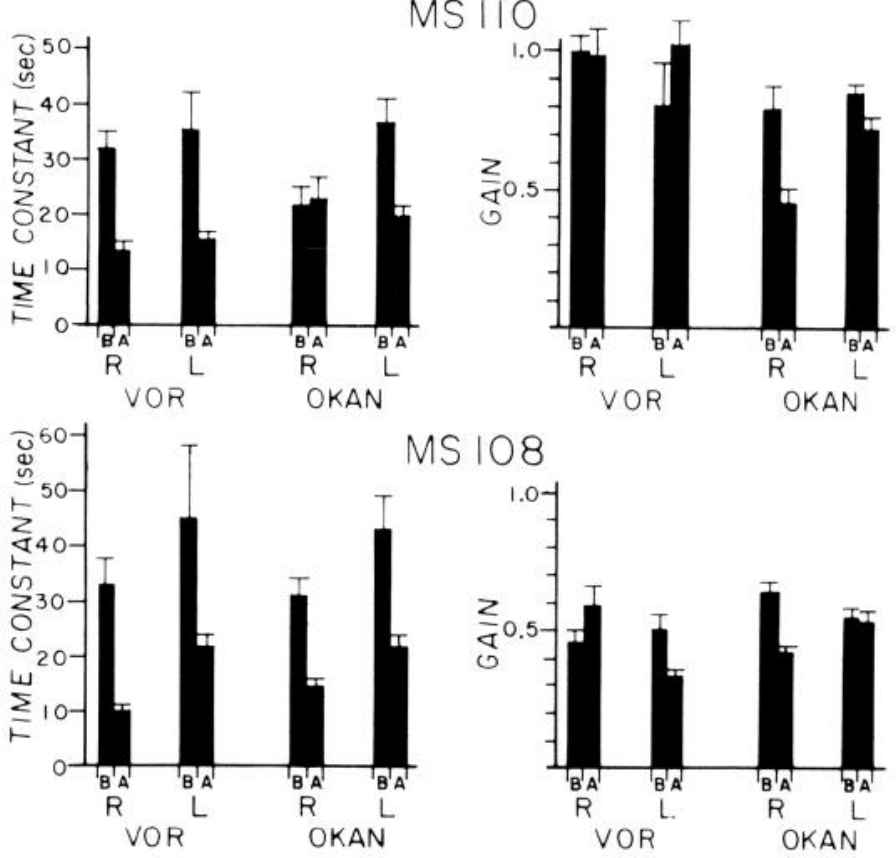

MS 108

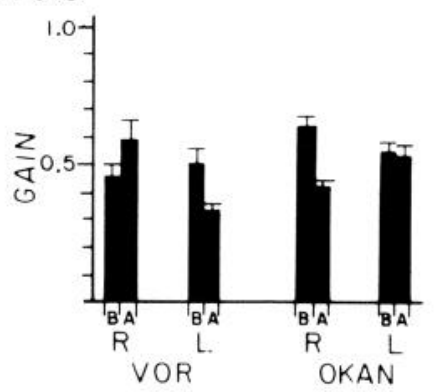

CONDITIONS

Figure 4. Bar graphs indicating the time constant and gain of post-rotatory nystagmus (VOR) and optokinetic afternystagmus (OKAN) before $(B)$ and after $(A) 20$ spins of the monkey's body to habituate VOR in a Ganzfeld. Direction of the slow phase of each type of nystagmus is labeled $R$ for right and $L$ for left. MS107, MS110, and MS108 identify each of the three monkeys who served as subjects. Error bars show one standard error above the parameter.

the spin was $80^{\circ}$ arc/sec. When it was clear that vestibular nystagmus had habituated, a second pair of left and right OKAN records were made and the session was terminated. Each session was separated from the previous one by 2 or more days to allow the effect of a session to dissipate. Each spin of the animal or drum was separated from the prior one by a period greater than $1 \mathrm{~min}$ to allow all aftereffects to subside.

The second protocol consisted of habituating the optokinetic reflex and testing for a change in vestibular nystagmus. The first optokinetic habituation session was separated from the last vestibular habituation by a period of at least 1 week. The time constants for post-rotatory nystagmus had recovered substantially for all monkeys. Here there were two sessions for each subject and each began with a left and right record of per- and postrotatory nystagmus. There followed 20 to 40 habituating 
exposures to optokinetic stimulation in alternating directions. Finally, a second pair of vestibular nystagmus records was obtained. Sessions again were separated by 2 or more days. All other conditions were the same as in protocol 1. During all tests in both protocols, random presentation of novel, somatosensory, and auditory stimulations were used to maintain the subjects' alertness.

Finally, a control session was obtained for each animal to show the effect of the temporal separation of our tests in the absence of habituating stimulation. Pairs of vestibular and optokinetic nystagmus records were obtained at the beginning and end of the session as befere. Then we departed from the habituation protocol by allowing the animal to spend about $1 \mathrm{hr}$ looking around the normally illuminated laboratory environment with its head restrained. There were no significant changes in the time constant for any animal under these conditions. Statistical reliability of the change of time constant was determined by a two-tailed $t$ test of the difference in the slopes of the regression coefficients determined before and after habituating exposure.

\section{Results}

Reduced persistence of post-rotatory vestibular nystagmus occasionally was accompanied by reduced persistence of optokinetic afternystagmus. Table I shows the time constants of post-rotatory nystagmus and OKAN before and after the habituating spins for all sessions of each of the monkeys. These data support two points.

First, the program of spins and stops reliably produced a symmetrical reduction in the time constant for persistence of slow phase velocity of post-rotatory nystagmus. In general, the time constant of post-rotatory nystagmus exceeded $30 \mathrm{sec}$ during the first pair of spins and had dropped to about one-third or less of its starting value by the end of the third habituation session. Seventy-eight percent of these decreases were statistically reliable ( $p$ $\leqslant 0.01)$.

Secondly, the persistence of optokinetic afternystagmus, i.e., the OKAN, usually decreased with that of postrotatory nystagmus ( $78 \%$ of the pairs). The time constants for OKAN were typically about $30 \mathrm{sec}$ or longer when first measured in these monkeys and they dropped to around one-half or less of their starting value by the end of the third vestibular habituation session. However, $64 \%$ of the decreases were not statistically reliable $(p \leqslant$ 0.05 ) and there were four tests on which the OKAN time constant increased after vestibular habituation.

The coupling of the changes in the time constant of the two kinds of responses can be seen more readily in Figure 3 which summarizes all of the data from this experiment. Each point shows the time constant of OKAN plotted against the time constant of post-rotatory nystagmus for a particular direction of slow eye velocity and habituation state. Although there is a trend for the OKAN time constant to be coupled to that of vestibular nystagmus, the relation was rarely tight as can be seen by the large scatter of the data points of MS107 and MS110. In fact, correlation coefficients for the data in Figure 3 were 0.27 for monkey MS107, 0.87 for MS108, and 0.25 for MS110.

Bar graphs illustrating the time constants and gains for both types of nystagmus before and after habituation are shown in Figure 4 for representative sessions of each of the monkeys. These graphs show that the gain of both vestibular nystagmus and OKAN was largely independent of the time constant of the nystagmus. Gains typically fell in the range 0.4 to 0.8 . This means that we were successful in maintaining alertness of the animals throughout all experiments.

Reducing the persistence of optokinetic afternystagmus did not produce reliable changes in the persistence of vestibular nystagmus. Table II shows the time con-

TABLE II

Time constants of horizontal optokinetic afternystagmus (OKAN) and post-rotatory vestibular nystagmus (PRN) before and after three monkeys were subjected to 20 habituating spins of an optokinetic drum in each of two sessions

\begin{tabular}{|c|c|c|c|c|c|c|c|c|}
\hline \multirow{2}{*}{ Monkey } & \multirow{2}{*}{ Session } & \multirow{2}{*}{$\begin{array}{l}\text { Direction of } \\
\text { Slow Phase } \\
\text { Eye Velocity }\end{array}$} & \multicolumn{3}{|c|}{ OKAN Time Constant ${ }^{a}$} & \multicolumn{3}{|c|}{ PRN Time Constant ${ }^{\alpha}$} \\
\hline & & & Before & & After & Before & & After \\
\hline \multirow{5}{*}{ MS107 } & & & & sec & & & $\sec$ & \\
\hline & 1 & Right & $-16.7(0.024)$ & $*$ & $-9.3(0.029)$ & $-19.2(0.007)$ & NS & $-14.1(0.013)$ \\
\hline & & Left & $-21.3(0.006)$ & NS & $-19.0(0.011)$ & $-35.7(0.006)$ & NS & $-23.6(0.007)$ \\
\hline & 2 & Right & $-18.8(0.016)$ & * & $-9.8(0.010)$ & $-17.9(0.012)$ & NS & $-14.4(0.007)$ \\
\hline & & Left & $-36.2(0.014)$ & * & $-11.7(0.011)$ & $-28.7(0.003)$ & ${ }^{*}$ & $-18.6(0.009)$ \\
\hline \multirow[t]{4}{*}{ MS108 } & 1 & Right & $-12.7(0.005)$ & * & $-6.5(0.013)$ & $-13.4(0.007)$ & * & $-9.9(0.010)$ \\
\hline & & Left & $-10.7(0.006)$ & * & $-6.6(0.013)$ & $-8.9(0.007)$ & * & $-13.6(0.004)$ \\
\hline & 2 & Right & $-6.1(0.012)$ & NS & $-7.5(0.018)$ & $-8.9(0.009)$ & NS & $-11.6(0.014)$ \\
\hline & & Left & $-8.7(0.020)$ & $*$ & $-6.7(0.023)$ & $-13.2(0.007)$ & ${ }^{*}$ & $-7.4(0.01)$ \\
\hline \multirow[t]{4}{*}{ MS110 } & 1 & Right & $-23.0(0.010)$ & $*$ & $-12.2(0.006)$ & $-31.1(0.004)$ & * & $-48.6(0.003)$ \\
\hline & & Left & $-36.1(0.002)$ & * & $-17.7(0.005)$ & $-71.6(0.005)$ & NS & $-39.1(0.005)$ \\
\hline & 2 & Right & $-17.5(0.010)$ & NS & $-15.2(0.005)$ & $-36.2(0.005)$ & NS & $-50.8(0.002)$ \\
\hline & & Left & $-32.7(0.002)$ & $*$ & $-15.7(0.003)$ & $-57.5(0.002)$ & ${ }^{*}$ & $-33.0(0.002)$ \\
\hline
\end{tabular}

${ }^{a}$ Log standard error of the slope from which the time constant was computed (reciprocal of slope) is shown in parentheses. Other features are the same as in Table I. 
stants of post-rotatory nystagmus and OKAN before and after habituating optokinetic stimulation. Repetitive alternating exposure to the spinning drum and Ganzfeld, which was used to induce OKN and OKAN in alternating directions, always produced a symmetrical reduction in the time constant of OKAN (statistically reliable in $75 \%$ of the cases). In fact, 201 -min exposures to the spinning drum were sufficient to halve OKAN time constants in Table II. However, these changes did not influence the persistence of vestibular nystagmus systematically. The nine tests which show a statistically reliable decrease in the OKAN time constant were accompanied by only four cases of statistically reliable decreases in the post-rota-
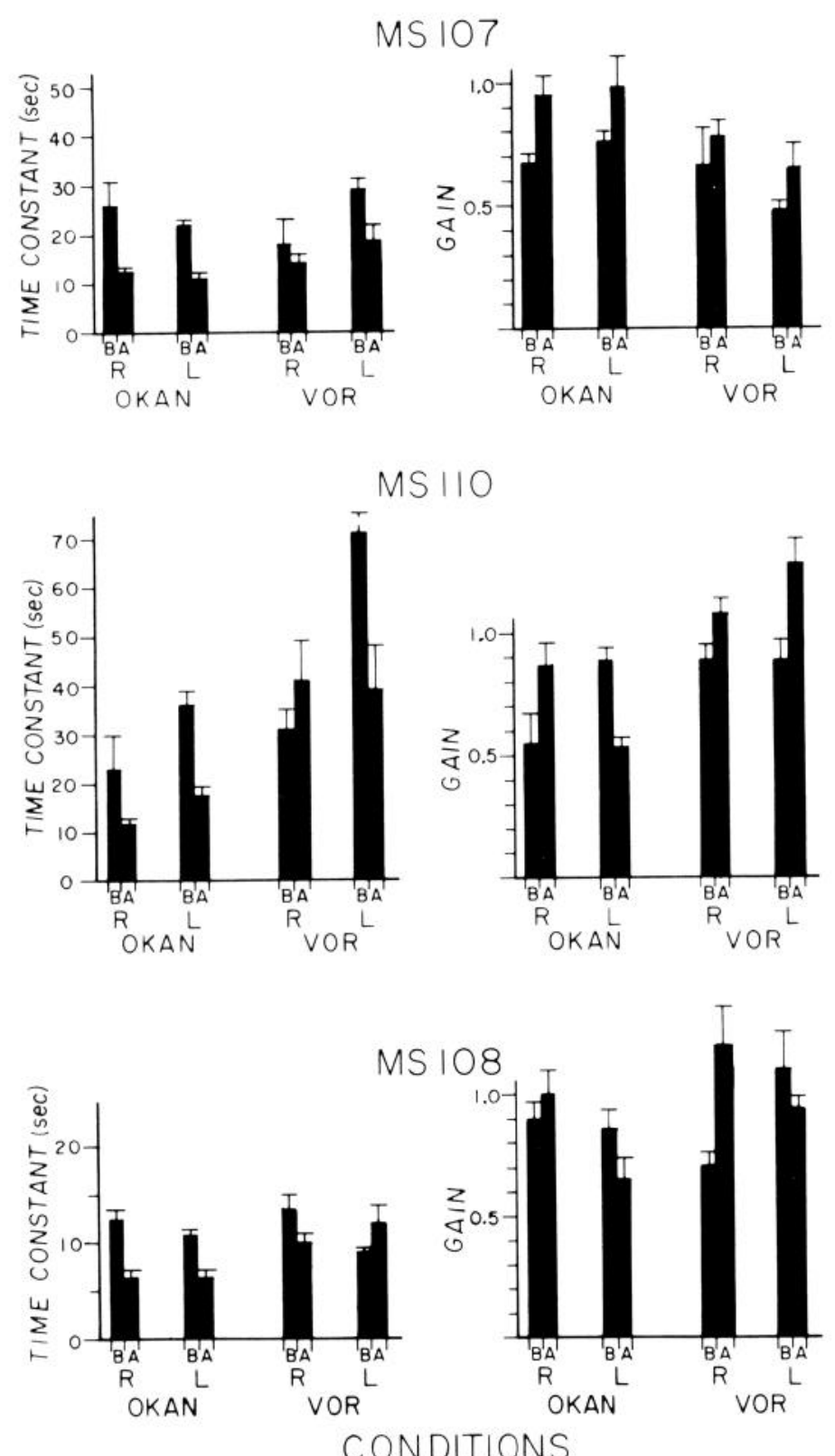

Figure 5. Bar graphs indicating the time constant and gain of optokinetic afternystagmus (OKAN) and post-rotatory nystagmus before $(B)$ and after $(A) 20$ spins of a patterned drum to habituate optokinetic afternystagmus. Other features are the same as in Figure 3. tory nystagmus time constant and there were two tests on which the latter time constants showed a statistically reliable increase.

Time constants and gains of both OKAN and postrotatory vestibular nystagmus are shown for representative sessions of each monkey in Figure 5. The bar graphs show that gains were again reasonably uniform, indicating that changing states of alertness had not contaminated the results.

\section{Discussion}

The present results replicate the observation of Blair and Gavin (1979) that a repetitive pattern of head angular accelerations produced a symmetrical decrease in persistence of vestibular nystagmus that was not due to a gain change or a systematic bias. Also, we have found here that a repetitive program of optokinetic stimulations reduced the persistence of OKAN without a change of its gain or bias. The absence of oculomotor bias (a systematic drift of the eyes) is of particular interest to the problem posed here. Changes in the optokinetic response previously reported to accompany habituation of vestibular nystagmus were based on unidirectional stimulation (Collins, 1974). This stimulation introduced a systematic bias (e.g., see Mowrer, 1935; Young and Henn, 1976) that interacted with all of the nystagmus tests and did not allow the clear conclusion that an actual change in the time constant of the nystagmus had occurred. In the present experiments, the procedure for fitting exponentials to slow phase eye velocity is insensitive to bias.

Our observation that we could frequently force a large change in the time constant of one kind of nystagmus (post-rotatory nystagmus or optokinetic afternystagmus) without a commensurate change in the time constant for the other kind is not compatible with the hypothesis that both kinds of nystagmus share a common velocity storage mechanism, but this must be tempered by the following considerations.

We have assumed that the modification of the time constant of nystagmus is gradual and will not change abruptly with the switch from optokinetic to vestibular stimulation or vice versa. This assumption seems secure because typically five to ten habituating spins are required to produce a measurable change in the nystagmus time constant on a naive animal. Furthermore, we have found that, once the VOR time constant has been habituated to a short value, $1 \mathrm{hr}$ of forced sinusoidal rotation of the animal's head with the room visible or sitting still looking around the room causes no significant changes in that time constant. In fact, at least $24 \mathrm{hr}$ exposure to natural body movement in the animal's home cage is necessary for any noticeable return of the time constant to its previous higher value. Hence, it seems unlikely that the time constant could change abruptly upward from one trial to the next with the change in type of stimulation.

We also do not feel that the failure to observe close coupling of the time constants of VOR and OKAN is a consequence of the sometimes capricious nature of EOG recording. In every instance in which we repeatedly stimulated one type of nystagmus, we always observed a 
decrease in the time constant for that type of nystagmus regardless of its initial value. Such changes were accompanied only occasionally by a decrease in the time constant for the other type of nystagmus. This observation suggests that our result is not a consequence of recording artifacts or the protocol used.

Our results then are not compatible with a single velocity storage mechanism for the two types of nystagmus. However, it would be an oversimplification to postulate two separate, independent storage mechanisms because, in at least one condition, VOR habituation in monkey MS108, Table I and Figure 4, there was fairly good coupling of the time constants of VOR and OKAN. The most acceptable approach under the circumstances is to postulate that there are regenerative neuronal circuit elements in the brain stem which can act to sustain a smooth eye movement once it has been initiated by the appropriate vestibular or optokinetic stimulus. The circuit elements are probably of the same kind for the two classes of movement and very likely in contiguous locations, with some interdigitation. Sherrington's diagram of partially overlapping neuron pools, used to explain "reflex occlusion," can be looked at as a prototype of what may be involved in this situation.

\section{References}

Blair, S., and M. Gavin (1979) Response of the vestibulo-ocular reflex to differing programs of acceleration. Invest. Ophthalmol. Vis. Sci. 18: 1086-1093.

Buettner, U., U. Büttner, and V. Henn (1978) Transfer characteristies of neurons in vestibular nuclei of the alert monkey. J. Neurophysiol. 41: 1614-1628.

Cohen, B., T. Uemura, and S. Takemori (1973) Effects of labyrinthectomy on optokinetic nystagmus (OKN) and optokinetic after-nystagmus (OKAN). Equilibrium Res. 3: 8893.
Cohen, B., U. Matsuo, and T. Raphan (1977) Quantitative analysis of the velocity characteristics of optokinetic nystagmus and optokinetic after-nystagmus. J. Physiol. (Lond.) 270: 321-344.

Collewijn, H. (1972) An analog model of the rabbit's optokinetic system. Brain Res. 36: 71-88.

Collins, W. E. (1974) Habituation of vestibular responses with and without visual stimulation. In Handbook of Sensory Physiology. Vol. VI/2: Vestibular System Part 2, H. Kornhuber, ed., pp. 369-388, Springer-Verlag, Berlin.

Fernandez, C., and J. Goldberg (1971) Physiology of peripheral neurons innervating semicircular canal of the squirrel monkey. II. Response to sinusoidal stimulation and dynamics of peripheral vestibular system. J. Neurophysiol. 34: 661-675.

Mowrer, O. H. (1935) Some neglected factors which influence the duration of post-rotational nystagmus. Acta Otolaryngol. (Stockh.) 22: 1-23.

Raphan, T., B. Cohen, and V. Matsuo (1977) A velocity-storage mechanism responsible for optokinetic nystagmus (OKN), optokinetic afternystagmus (OKAN) and vestibular nystagmus. In: Control of Gaze by Brain Stem Neurons, R. Baker and A. Bethoz, eds., pp. 37-47, Elsevier-North Holland, Amsterdam and New York.

Robinson, D. A. (1977a) Vestibular and optokinetic symbioses: An example of explaining by modelling. In Control of Gaze by Brain Stem Neurons, R. Baker and A. Bertoz, eds., pp. 49-58, Elsevier-North Holland, Amsterdam and New York.

Robinson, D. A. (1977b) Linear addition of optokinetic and vestibular signals in the vestibular nucleus. Exp. Brain Res. 30: $447-450$.

Skavenski, A. A., and D. A. Robinson (1973) Role of abducens neurons in vestibuloocular reflex. J. Neurophysiol. 36: 724738.

Waespe, W., and V. Henn (1977) Neuronal activity in the vestibular nuclei of the alert monkey during vestibular and optokinetic stimulation. Exp. Brain Res. 27: 523-538.

Young, L. R., and V. S. Henn (1976) Selective habituation of vestibular nystagmus by visual stimulation in the monkey. Acta Otolaryngol. 82: 165-171. 